\title{
Critical parameter equations for degenerate parabolic equations coupled via nonlinear boundary flux
}

\author{
Si $\mathrm{Xu}^{*}$ and Zifen Song
}

* Correspondence: xusi_math@hotmail.com Department of Mathematics, Jiangxi Vocational College of Finance and Economics, Jiujiang, Jiangxi, 332000, PR China

\section{Abstract}

This paper deals with the critical parameter equations for a degenerate parabolic system coupled via nonlinear boundary flux. By constructing the self-similar supersolution and subsolution, we obtain the critical global existence parameter equation. The critical Fujita type is conjectured with the aid of some new results. Mathematics Subject Classification (2000). 35K55; 35K57.

Keywords: degenerate parabolic system, global existence, blow-up

\section{Introduction}

In this paper, we consider the following degenerate parabolic equations

$$
\frac{\partial u_{i}}{\partial t}=\left(u_{i}^{p_{i}}\right)_{x x}, \quad(i=1,2, \ldots, k), \quad x>0,0<t<T,
$$

coupled via nonlinear boundary flux

$$
-\left(u_{i}^{p_{i}}\right)_{x}(0, t)=u_{i+1}^{q_{i+1}}(0, t), \quad(i=1,2, \ldots, k), u_{k+1}:=u_{1}, q_{k+1}:=q_{1} \quad 0<t<T,(1,2
$$

with continuous, nonnegative initial data

$$
u_{i}(x, 0)=u_{0 i}(x), \quad(i=1,2, \ldots, k), \quad x>0,
$$

compactly supported in $\mathbb{R}_{+}$, where $p_{i}>1, q_{i}>0,(i=1,2, \ldots, k)$ are parameters.

Parabolic systems like (1.1)-(1.3) appear in several branches of applied mathematics. They have been used to models, for example, chemical reactions, heat transfer, or population dynamics (see [1] and the references therein).

As we shall see, under certain conditions the solutions of this problem can become unbounded in a finite time. This phenomenon is known as blow-up, and has been observed for several scalar equations since the pioneering work of Fujita [2]. For further references, see the review by Leivine [3]. Blow-up may also happen for systems (see [4-7]). Our main interest here will be to determine under which conditions there are solutions of (1.1)-(1.3) that blow up and, in the blow-up case, the speed at which blowup takes place, and the localization of blow-up points in terms of the parameters $p_{i}, q_{i},(i=1,2, \ldots, k)$.

As a precedent, we have the work of Galaktionov and Levine [8], where they studied the single equation

(c) 2011 Xu and Song; licensee Springer. This is an Open Access article distributed under the terms of the Creative Commons Attribution License (http://creativecommons.org/licenses/by/2.0), which permits unrestricted use, distribution, and reproduction in any medium, provided the original work is properly cited. 


$$
\begin{aligned}
& u_{1 t}=\left(u_{1}^{p_{1}}\right)_{x x}, \quad x>0,0<t<T, \\
& -\left(u_{1}^{p_{1}}\right)_{x}(0, t)=u_{1}^{q_{2}}(0, t), \quad 0<t<T, \\
& u_{1}(x, 0)=u_{01}(x), \quad x>0 .
\end{aligned}
$$

It was shown if $0<q_{2} \leq q_{0}=\left(p_{1}+1\right) / 2$, then all nonnegative solutions of (1.4) are global in time, while for $q_{2}>q_{0}$ there are solutions with finite time blow-up. That is, $q_{0}$ is the critical global existence exponent. Moreover, it was shown that $q_{c}:=p_{1}+1$ is a critical exponent of Fujita type. Precisely, $q_{c}$ has the following properties: if $q_{0}<q_{2} \leq q_{c}$, the all nontrivial nonnegative solutions blow up in a finite time, while global nontrivial nonnegative solutions exist if $q_{2}>q_{c}$.

We remark that there are some related works on the critical exponents for (1.1)-(1.3) in special cases.

In [9-11], the authors consider the case for $p_{i}=1,(i=1,2, \ldots, k)$.

In [12], the authors consider the case for $k=2$.

For the system (1.1)-(1.3), instead of critical exponents there are critical parameter equations, one for global existence and another of Fujita type. This is the content of our first theorem.

To state our results, we introduce some useful symbols. Denote by

$$
A=\left(\begin{array}{cccccccc}
1+p_{1} & -2 q_{2} & 0 & 0 & \cdots & 0 & 0 & 0 \\
0 & 1+p_{2} & -2 q_{3} & 0 & \cdots & 0 & 0 & 0 \\
\cdots & \cdots & \cdots & \cdots & \cdots & \cdots & \cdots & \cdots \\
0 & 0 & 0 & 0 & \cdots & 0 & 1+p_{k-1} & -2 q_{k} \\
-2 q_{1} & 0 & 0 & 0 & \cdots & 0 & 0 & 1+p_{k}
\end{array}\right)
$$

A series of standard computations yield

$$
\operatorname{det} A=\prod_{l=1}^{k}\left(1+p_{l}\right)-\prod_{l=1}^{k} 2 q_{l} .
$$

We shall see that $\operatorname{det} A=0$ is the critical global existence parameter equation. Let $\left(\alpha_{1}, \alpha_{2}, \ldots, \alpha_{k}\right)^{T}$ be the solution of the following linear algebraic system

$$
A\left(\alpha_{1}, \alpha_{2}, \ldots, \alpha_{k-1}, \alpha_{k}\right)^{T}=(1,1, \ldots, 1,1)^{T},
$$

that is

$$
\alpha_{i}=\frac{\prod_{l=1}^{k}\left(1+p_{l}\right)}{2 q_{i}\left[\prod_{l=1}^{k}\left(1+p_{l}\right)-\prod_{l=1}^{k} 2 q_{l}\right]} \sum_{m=i}^{k+i-1} \prod_{j=i}^{m} \frac{2 q_{j}}{1+p_{j}}, q_{k+i}=q_{i}, p_{k+i}=p_{i}(i=1,2, \cdots, k) .
$$

We define

$$
\beta_{i}=\frac{1+\left(p_{i}-1\right) \alpha_{i}}{2}, \quad(i=1,2, \cdots, k) .
$$

\section{Theorem 1.1.}

(I) If $\prod_{l=1}^{k}\left(1+p_{l}\right) \geq \prod_{l=1}^{k} 2 q_{l}($ i.e. $\operatorname{det} A \geq 0)$, every nonnegative solution of (1.1)-(1.3) is global in time.

(II) If $\prod_{l=1}^{k}\left(1+p_{l}\right)<\prod_{l=1}^{k} 2 q_{l}$ (i.e. $\left.\operatorname{det} A<0\right)$ and there exists $j(1 \leq j \leq k)$ such that $\alpha_{j}$ $+\beta_{j} \leq 0$, then every nonnegative, nontrivial solution blows up in finite time. 
(III) If $\prod_{l=1}^{k}\left(1+p_{l}\right)<\prod_{l=1}^{k} 2 q_{l}(i . e . \operatorname{det} A<0)$, with $\alpha_{i}+\beta_{i}>0(i=1,2, \ldots, k)$, there exist nonnegative solutions with blow-up and nonnegative solutions that are global.

Therefore, the critical global existence parameter equation is

$$
\prod_{l=1}^{k}\left(1+p_{l}\right)=\prod_{l=1}^{k} 2 q_{l}(\text { i.e. } \operatorname{det} A=0)
$$

and the critical Fujita type parameter equation is

$$
\min \left\{\alpha_{1}+\beta_{1}, \alpha_{2}+\beta_{2}, \ldots, \alpha_{k}+\beta_{k}\right\}=0 .
$$

The values of $\alpha_{i}, \beta_{i}(i=1,2, \ldots, k)$ are the exponents of self-similar solutions to problem (1.1)-(1.2). Such self-similar solutions are studied in Section 2, and play an important role in the proof of Theorem 1.1.

Let us observe that if we take $k=2$, the critical parameter equations coincide with those found in [12].

The rest of this paper is organized as follows. In the next section, we study the existence of self-similar solutions of different type. In Section 3 we give some results concerning existence, comparison, monotonicity and uniqueness. In Section 4 we find the critical parameter equations (Theorem 1.1).

\section{Self-similar solutions}

In this section, we consider different kinds of self-similar solutions of problem (1.1)(1.2). We have the following results.

Theorem 2.1. Let

$$
u_{i}(x, t)=(T-t)^{\alpha_{i}} f_{i}\left(\xi_{i}\right), \quad \xi_{i}=x(T-t)^{-\beta_{i}}, i=1,2, \ldots, k .
$$

If

$$
\prod_{l=1}^{k}\left(1+p_{l}\right)<\prod_{l=1}^{k} 2 q_{l}
$$

there is a self-similar solution of problem (1.1)-(1.2) blowing up in a finite time $T>0$, of form (2.1). Moreover, the support of $f_{i}$ is $\mathbb{R}_{+}$if $\beta_{i}>0$, and a compact set if $\beta_{i} \leq 0$ ( $i=$ $1,2, \ldots, k)$.

Theorem 2.2. Let

$$
u_{i}(x, t)=t^{\alpha_{i}} f_{i}\left(\xi_{i}\right), \xi_{i}=x t^{-\beta_{i}}, i=1,2, \ldots, k .
$$

(a) If

$$
\prod_{l=1}^{k}\left(1+p_{l}\right)>\prod_{l=1}^{k} 2 q_{l},
$$

then there exist functions $f_{i}$ positive in $\mathbb{R}_{+}$, such that $u_{i}$ given in (2.3) is a self-similar solution of problem (1.1)-(1.2) global in time. These solutions have $\alpha_{i}>0$ and thus their initial data are identically zero. Then $\beta_{i}<0(i=1,2, \ldots, k)$.

(b)If

$$
\alpha_{i}+\beta_{i}>0, \quad i=1,2, \ldots, k,
$$


then there exist functions $f_{i j}$ compactly supported in $\mathbb{R}_{+}$, such that $u_{i}$ given in (2.3) is a self-similar solution of problem (1.1)-(1.2) global in time. These solutions have $\alpha_{i}<0$ and thus they decay to zero as $t \rightarrow \infty$. Then $\beta_{i}>0$, and hence their supports expand as time increases.

Remark 2.2. If there exists $j(1 \leq j \leq k)$ such that $\alpha_{j}+\beta_{j} \leq 0$, there are no profiles $f_{i}$ $\in L^{1}\left(\mathbb{R}_{+}\right)$such that $u_{i}(i=1,2, \ldots, k$, given by (2.3) is a solution. Indeed

$$
\int_{0}^{\infty} u_{j}(x, t) d x=t^{\alpha_{j}+\beta_{j}} \int_{0}^{\infty} f_{j}\left(\xi_{j}\right) d \xi_{j} .
$$

Then, if $\alpha_{j}+\beta_{j} \leq 0$, the mass of $u_{j}$ would not increase, a contradiction.

Theorem 2.3. Let

$$
u_{i}(x, t)=e^{\alpha_{i} t} f_{i}\left(\xi_{i}\right), \quad \xi_{i}=x e^{-\beta_{i} t}, \quad i=1,2, \ldots, k .
$$

If

$$
\prod_{l=1}^{k}\left(1+p_{l}\right)=\prod_{l=1}^{k} 2 q_{l}
$$

for any $\alpha_{1}>0$, there is a self-similar solution of problem (1.1)-(1.2) global in time of form (2.5) where

$$
\alpha_{i}=\alpha_{1} \prod_{j=2}^{i} \frac{1+p_{j-1}}{2 q_{j}}(i=2, \ldots, k), \quad \beta_{i}=\frac{\left(p_{i}-1\right) \alpha_{i}}{2}(i=1,2, \ldots, k) .
$$

Moreover, the supports of $f_{i}(i=1,2, \ldots, k)$ are compact.

Remark 2.3. The solutions are in principle weak. However, if they are positive everywhere, they are also classical.

In order to prove these theorems, we will use the following results of Gilding and Peletier (see [13-15]):

Theorem 2.4. Let $a, b, V \in \mathbb{R}$ and $U \geq 0$. For fixed $a$ and $b$, let $S_{A}$ denote the set of values of $(U, V)$ such that there exists a weak, nonnegative, compactly supported solution $f_{1}$ of

$$
\begin{aligned}
& \left(f_{1}^{p_{1}}\right)^{\prime \prime}(\eta)+a \eta f_{1}^{\prime}(\eta)=b f_{1}(\eta), \quad 0<\eta<\infty, \\
& f_{1}(0)=U, \\
& \left(f_{1}^{p_{1}}\right)^{\prime}(0)=V,
\end{aligned}
$$

and let $S_{B}$ denote the set of values $(U, V)$ for which there exists a bounded, positive, classical solution $f_{1}$ of (2.8)-(2.10).

(a) If $b<0$ and $2 a+b<0$, then $S_{A}=\{(0,0)\}$ and $S_{B}=\varnothing$.

(b) If $b<0$ and $2 a+b=0$, then $S_{A}=\{(0, V): 0 \leq V<\infty\}$ and $S_{B}=\varnothing$.

(c) If $b \leq 0$ and $2 a+b>0$, then there exists a unique $V$ * such that $S_{A}=\left\{\left(U, U^{\left(p_{1}+1\right) / 2} V_{*}\right): 0 \leq U<1\right\}$ and $S{ }_{B}=\{(U, V): 0 \leq U<\infty$, $\left.U^{\left(p_{1}+1\right) / 2} V_{*}<V<\infty\right\}$, where $V_{*}>0$ if $a+b<0, V_{*}=0$ if $a+b=0$, and $V_{*}<0$ if $a+$ $b>0$. 
(d) If $b>0$ and $a \geq 0$, then there exists a unique $V_{*}<0$ such that $S_{A}=\left\{\left(U, U^{\left(p_{1}+1\right) / 2} V_{*}\right): 0 \leq U<1\right\}$ and $S_{B}=\varnothing$.

(e) If $b>0$ and $a<0$, or $b=0$ and $a \leq 0$, then $S_{A}=\{(0,0)\}$ and there exists $a$ unique $V_{*}$ such that $S_{B}=\left\{\left(U, U^{(p 1+1) / 2} V_{*}\right): 0 \leq U<\infty\right\}$, where $V_{*}<0$ if $b>0$ and $V_{*}=$ 0 if $b=0$.

Moreover, for each $(U, V) \in S_{A} \cup S_{B}$ there exists at most one weak solution of (2.8)(2.10).

Remark 2.4. In the case where $a=\left(\left(p_{1}-1\right) / 2\right) b>0$, we have $V_{*}=-1$. This is a consequence of the existence for a self-similar solution of exponential form for the scalar problem (1.4) with $q_{2}=\left(p_{1}+1\right) / 2$ (see [8]).

Proof of Theorem 2.1. We consider solutions of form (2.1). Imposing that the porous equations (1.1) are fulfilled, we get the following relations for the parameters:

$$
\alpha_{i}-1=\alpha_{i} p_{i}-2 \beta_{i}, \quad i=1,2, \ldots, k .
$$

On the other hand, the boundary conditions (1.2) imply that

$$
\alpha_{i} p_{i}-\beta_{i}=\alpha_{i+1} q_{i+1}, \quad i=1,2, \ldots, k, \alpha_{k+1}=\alpha_{1}, \quad q_{k+1}=q_{1} .
$$

Solving the linear systems (2.11)-(2.12), we get that $\alpha_{i}, \beta_{i}(i=1,2, \ldots, k)$ are given by (1.5) and (1.6). Therefore, $\alpha_{i}<0(i=1,2, \ldots, k)$ if and only if $\prod_{l=1}^{k}\left(1+p_{l}\right)<\prod_{l=1}^{k} 2 q_{l}$. On the other hand, the profiles must satisfy

$$
\left(f_{i}^{p_{i}}\right)^{\prime \prime}\left(\xi_{i}\right)-\beta_{i} \xi_{i} f_{i}^{\prime}\left(\xi_{i}\right)=-\alpha_{i} f_{i}\left(\xi_{i}\right), \quad i=1,2, \ldots, k,
$$

plus the boundary conditions

$$
-\left(f_{i}^{p_{i}}\right)^{\prime}(0)=f_{i+1}^{q_{i+1}}(0), \quad i=1,2, \ldots, k, \quad q_{k+1}=q_{1}, f_{k+1}=f_{1} .
$$

Then $f_{i}$ satisfy (2.8) with coefficients $a_{i}=-\beta_{I}, b_{i}=-\alpha_{i}(i=1,2, \ldots, k)$. Thus, Theorem 2.4 parts (d) and (e) says that there is an one-parameter family (parameter $U_{i}$ ) of (2.8) satisfying

$$
f_{i}(0)=U_{i,} \quad\left(f_{i}^{p_{i}}\right)^{\prime}(0)=U_{i}^{\left(p_{i}+1\right) / 2} V_{* i},
$$

where $V_{* i}<0(i=1,2, \ldots, k)$ are constants. The profile $f_{i}$ has compact support if $\beta_{i} \leq$ 0 and is positive in $\mathbb{R}_{+}$if $\beta_{i}>0$. We choose $U_{i}$ such that the boundary conditions (2.14) are fulfilled, that is

$$
-U_{i}^{\left(p_{i}+1\right) / 2} V_{* i}=U_{i+1}^{q_{i+1}}, \quad i=1,2, \ldots, k, \quad U_{k+1}=U_{1}, q_{k+1}=q_{1} .
$$

Taking logarithms, this is equivalent to

$$
A\left(\ln U_{1}, \ln U_{2}, \ldots, \ln U_{k-1}, \ln U_{k}\right)^{T}=-2\left(\ln \left|V_{* 1}\right|, \ln \left|V_{* 2}\right|, \ldots, \ln \left|V_{* k-1}\right|, \ln \left|V_{* k}\right|\right)^{T} .
$$

As $\prod_{l=1}^{k}\left(1+p_{l}\right) \neq \prod_{l=1}^{k} 2 q_{l}$ (i.e. $\left.\operatorname{det} A \neq 0\right)$, the above system has a unique solution. $\square$

Proof of Theorem 2.2. We are considering solutions of the form (2.3). Imposing that the equations (1.1) and that boundary conditions (1.2) are fulfilled, we get that the exponents should satisfy the relations (2.11)-(2.12). Hence they are given by (1.5)-(1.6). Moreover, the boundary conditions for the profiles are given by (2.14). However, the equations for the profiles are now different: 


$$
\left(f_{i}^{p_{i}}\right)^{\prime \prime}\left(\xi_{i}\right)+\beta_{i} \xi_{i} f_{i}^{\prime}\left(\xi_{i}\right)=\alpha_{i} f_{i}\left(\xi_{i}\right), \quad i=1,2, \ldots, k
$$

Thus, $f_{i}$ satisfy (2.8) with coefficients $a_{i}=\beta_{i}, b_{i}=\alpha_{i}(i=1,2, \ldots, k)$.

(I) If $\alpha_{i}>0$, that is, if (2.4) holds, then $\beta_{i}<0(i=1,2, \ldots, k)$. Therefore, applying Theorem 2.4 part (d) as in the proof of Theorem 2.1, and taking the solutions of (2.15) as values for parameters, we obtain that there exist positive profiles $f_{i}(i=1,2, \ldots, k)$ solving (2.16) and satisfying (2.14).

(II) If $\alpha_{i}<0$ and $\alpha_{i}+\beta_{i}>0(i=1,2, \ldots, k)$, we can apply Theorem 2.4 part (c) as in the proof of Theorem 2.1 and taking the solutions of (2.15) as the parameters, we obtain that there exist compactly supports profiles $f_{i}(i=1,2, \ldots, k)$ solving $(2.16)$ and satisfying the boundary conditions (2.14).

Proof of Theorem 2.3. We are considering solutions of the form (2.5). Though the boundary conditions (1.2) impose (2.12) again, now equations (1.1) impose different relations for the exponents. Namely

$$
\alpha_{i}=\alpha_{i} p_{i}-2 \beta_{i}, \quad i=1,2, \ldots, k
$$

Thus,

$$
A\left(\alpha_{1}, \alpha_{2}, \ldots, \alpha_{k-1}, \alpha_{k}\right)^{T}=(0,0, \ldots, 0,0)^{T} .
$$

There are nontrivial solutions of (2.18) if and only if $\prod_{l=1}^{k}\left(1+p_{l}\right)=\prod_{l=1}^{k} 2 q_{l}$ (i.e. det $A=0)$. In this case, $\beta_{1}, \alpha_{I}, \beta_{i}(i=2, \ldots, k)$ are related to $\alpha_{1}$ by $(2.7)$.

The boundary conditions for the profiles are again given by (2.14), while the equations for the profiles are given by (2.16). If $\alpha_{1}>0$, then $\beta_{1}, \alpha_{i}, \beta_{i}>0(i=2, \ldots, k)$ and $\beta_{i}=\left(\left(p_{i}-1\right) / 2\right) \alpha_{i}(i=1, \ldots, k)$. Hence, using Remark 2.4, we have solutions of (2.16) with $V_{* i}=-1(i=1,2, \ldots, k)$. Choosing one of the solutions of (2.15) with right-hand side zero (again we are using $\prod_{l=1}^{k}\left(1+p_{l}\right)=\prod_{l=1}^{k} 2 q_{l}$ (i.e. $\left.\operatorname{det} A=0\right)$ ), we obtain that there exist compactly supported profiles $f_{i}(i=1,2, \ldots, k)$ solving (2.16) and satisfying (2.14).

\section{Existence and uniqueness}

First, we state a theorem that guarantees the existence of a solution. It can be obtained using a standard monotonicity argument following ideas from [16].

Theorem 3.1. Given continuous, compactly supported initial data $u_{0 i}(x)(i=2, \ldots, k)$, there exists a local in time continuous weak solution of (1.1)-(1.3). Moreover, if the initial data are smooth and compatible in sense that

$$
-\left(u_{0 i}^{p_{i}}\right)_{x}(0)=u_{0 i+1}^{q_{i+1}}(0), \quad i=2, \ldots, k, \quad u_{0 k+1}(x)=u_{01}(x),
$$

then the solution has continuous time derivatives down to $t=0$.

Proof. Let us consider the Neumann problem

$$
\begin{aligned}
& w_{t}=\left(w^{r}\right)_{x x}, \quad x>0, \quad 0<t<\tau, \\
& -\left(w^{r}\right)_{x}(0, t)=h(t), \quad 0<t<\tau, \\
& w(x, 0)=w_{0}(x), \quad x>0,
\end{aligned}
$$

with $r>1$. We define the operator $M_{q_{i+1}}: C([0, \tau]) \rightarrow C([0, \tau])$ as $M_{q_{i+1}}(h)(t)=w^{q_{i+1}}(0, t)$, where $w(x, t)$ is the unique solution of (3.1) with $r=p_{i}$ and initial condition $w_{0}(x)=u_{0 i}(x)\left(i=1,2, \ldots, k, M_{q_{k+1}}=M_{q_{1}}, w^{q_{k+1}}=w^{q_{1}}\right)$. 
It has been proved in [17] that $M_{q_{i}}(i=1,2, \ldots, k)$ is continuous and compact. Moreover, they are order preserving.

Now let $A(h)=M_{q_{k}} \circ M_{q_{k-1}} \circ \cdots \circ M_{q_{2}} \circ M_{q_{1}}(h)$. Using the method of monotone iterations, one can prove that there exist $\tau>0$ such that $A$ has a fixed point in $C([0$, $\tau])$. This fixed point provides us with a continuous weak solution of (1.1)-(1.3) up to time $\tau$.

In order to obtain the regularity of the solution with compatible initial data, we only have to observe that the solution of (3.1) is regular if $-\left(w_{0}^{r}\right)_{x}=h(0)$ (see [18]).

Remark 3.1. If the initial data are compactly support, the solution $u_{i}(i=1,2, \ldots, k)$ also has compact support as long as it exists.

Remark 3.2. If the initial data are nontrivial, we can assume that they satisfy $u_{0 i}(x)>$ $0(i=1,2, \ldots, k)$. If not, $u_{i}(0, t)(i=1,2, \ldots, k)$ eventually become positive (compare with a Barenblatt solution of the corresponding equation).

Next, we define what called a subsolution and a supersolution for (1.1)-(1.2).

Definition 3.1. $\left(\underline{u}_{1}, \underline{u}_{2}, \ldots, \underline{u}_{k-1}, \underline{u}_{k}\right)$ is a subsolution of (1.1)-(1.2) if it satisfies

$$
\begin{aligned}
& \frac{\partial \underline{u}_{i}}{\partial t} \leq\left(u_{i}^{p_{i}}\right)_{x x} x>0,0<t<T, \quad i=1,2, \ldots, k, \\
& -\left(\underline{u}_{i}^{p_{i}}\right)_{x}(0, t) \leq \underline{u}_{i+1}^{q_{i+1}}(0, t), \underline{u}_{k+1}^{q_{k+1}}=\underline{u}_{1}^{q_{1}}, 0<t<T, \quad i=1,2, \ldots, k .
\end{aligned}
$$

Definition 3.2. We call $\left(\bar{u}_{1}, \bar{u}_{2}, \ldots, \bar{u}_{k-1}, \bar{u}_{k}\right)$ a supersolution of (1.1)-(1.2) of it satisfies (3.2)-(3.3) with the opposite inequalities.

With these definitions of super and subsolutions, we can state a comparison lemma.

Lemma 3.1 Let $\left(\bar{u}_{1}, \bar{u}_{2}, \ldots, \bar{u}_{k-1}, \bar{u}_{k}\right)$ be a supersolution and $\left(\underline{u}_{1}, \underline{u}_{2}, \ldots, \underline{u}_{k-1}, \underline{u}_{k}\right)$ be a subsolution. If

$$
\underline{u}_{i}(x, 0) \leq \bar{u}_{i}(x, 0), \quad i=1,2, \ldots, k,
$$

with

$$
\underline{u}_{i}(0,0) \leq \bar{u}_{i}(0,0), \quad i=1,2, \ldots, k,
$$

then

$$
\underline{u}_{i}(x, t) \leq \bar{u}_{i}(x, t), \quad i=1,2, \ldots, k,
$$

as long as both super and subsolutions exist.

Proof. It is standard, therefore we omit the details. Assume that the result is false. Let $t_{0}$ be the maximum time such that

$$
\underline{u}_{i}(x, t) \leq \bar{u}_{i}(x, t), i=1,2, \ldots, k,
$$

up to $t_{0}$. This time $t_{0}$ must be positive, by continuity. At that time, we must have $\underline{u}_{j}\left(0, t_{0}\right)=\bar{u}_{j}\left(0, t_{0}\right)$ for some $j(1 \leq j \leq k)$. Let us assume that $\underline{u}_{1}\left(0, t_{0}\right)=\bar{u}_{1}\left(0, t_{0}\right)$. Now the result follows by an application of Hopf's lemma. Indeed, $\bar{u}_{1}-\underline{u}_{1}$ satisfies a uniformly parabolic equation in a neighborhood of $x=0$, attains a minimum at $\left(0, t_{0}\right)$, and the corresponding flux is greater or equal than zero, a contradiction.

Now we state a lemma that guarantees that, for certain initial data, the solution of (1.1)-(1.3) increases in time. 
Lemma 3.2 Let $u_{0 i}(x)$ be the initial data for (1.1) -(1.3) such that $u_{0 i}(x)$ are smooth, satisfy the compatibility condition at the boundary and $\left(u_{0 i}^{p_{i}}\right)_{x x} \geq 0$. Then $u_{i}(x, t)$ increases in time, i.e., $u_{i t}(x, t) \geq 0(i=1,2, \ldots, k)$.

Proof. Let $w_{i}=u_{i t}$. Then, as the solutions are smooth (Theorem 3.1), we can differentiate to obtain the $\left(w_{1}, \ldots, w_{k}\right)$ is a solution of

$$
\begin{aligned}
& w_{i t}=\left(p_{i} u_{i}^{p_{i}-1} w_{i}\right)_{x x}, i=1, \quad 2, \ldots k, \\
& -\left(p_{i} u_{i}^{p_{i}-1} w_{i}\right)_{x}(0, t)=q_{i+1} u_{i+1}^{q_{i+1}-1} w_{i+1}(0, t), \quad q_{k+1}=q_{1}, \quad u_{k+1}=u_{1}, \quad w_{k+1}=w_{1},
\end{aligned}
$$

with initial data satisfying

$$
w_{i}(x, 0) \geq 0, \quad i=1,2, \ldots, k .
$$

To conclude the proof we apply the maximum principle. Due to the degeneration of the equations this cannot be done directly. A standard regularization procedure is needed (see [8] for details).

Next, we deal with the problem of uniqueness versus non-uniqueness for (1.1)-(1.3) on the case of vanishing initial data $\left(u_{0 i}(x)=0, i=1,2, \ldots, k\right)$.

Theorem 3.2

(a) Let $\prod_{l=1}^{k}\left(1+p_{l}\right)>\prod_{l=1}^{k} 2 q_{l}$. Then there exists a nontrivial solution with zero initial data that becomes positive at $x=0$ instantaneously. Then there is no uniqueness for problem (1.1)-(1.3) with zero initial data.

(b) Let $\prod_{l=1}^{k}\left(1+p_{l}\right) \leq \prod_{l=1}^{k} 2 q_{l}$. Then the solution of (1.1)-(1.3) with zero initial data is unique.

Proof.

(a) The self-similar solutions constructed in Theorem 2.2 become positive at $x=0$ instantaneously.

(b) We can construct small supersolution with the aid of the self-similar ones of exponential form that we found in Theorem 2.3. First, choose $\widetilde{q}_{1} \leq q_{1}$ such that $\bar{u}_{i}(x, t)=e^{\alpha_{i}(t+\tau)} f_{i}\left(x e^{-\beta_{i}(t+\tau)}\right), \quad i=1,2, \ldots, k$.

$$
\bar{u}_{i}(x, t)=e^{\alpha_{i}(t+\tau)} f_{i}\left(x e^{-\beta_{i}(t+\tau)}\right), \quad i=1,2, \ldots, k,
$$

where $\alpha_{1}>0$ is arbitrary and $\beta_{1}, \alpha_{i}, \beta_{i},(i=2, \ldots, k)$ are given by (2.7). Now we observe that $\left(\bar{u}_{1}, \bar{u}_{2}, \ldots, \bar{u}_{k-1}, \bar{u}_{k}\right)$ be a supersolution is a supersolution of (1.1)-(1.3) as long as $\bar{u}_{1}(0, t) \leq 1$. By the comparison Lemma 3.1, we obtain that every solution has initial data identically zero satisfies

$$
\bar{u}_{i}(x, t) \geq u_{i}(x, t), \quad i=1,2, \ldots, k .
$$

As $\bar{u}_{i}$ can be chosen as small as we want (using $\tau$ negative and large enough) we conclude that $\bar{u}_{i} \equiv 0(i=1,2, \ldots, k)$.

\section{Blow-up versus global existence}

We devote this section to prove Theorem 1.1. We borrow ideas from [8]. However, the fact that we are dealing with a system instead of a single equation forces us to develop a significantly different proof. We will organize the proof in several lemmas.

Our first lemma proves part (I) of Theorem 1.1. 
Lemma 4.1. If $\prod_{l=1}^{k}\left(1+p_{l}\right) \geq \prod_{l=1}^{k} 2 q_{l}(i . e . \operatorname{det} A \geq 0)$, every nonnegative solution of (1.1)-(1.3) is global in time.

Proof. It is enough to construct global supersolutions with initial data as large as needed. We achieve this with the aid of the self-similar solutions of exponential form that we found in Theorem 2.3.

First we choose $\widetilde{q}_{1} \geq q_{1}$ such that $2 \widetilde{q}_{1} \prod_{l=2}^{k} 2 q_{l}=\prod_{l=1}^{k}\left(1+p_{l}\right)$ and we let

$$
\bar{u}_{i}(x, t)=e^{\alpha_{i}(t+\tau)} f_{i}\left(x e^{-\beta_{i}(t+\tau)}\right), \quad i=1,2, \ldots, k,
$$

where $\alpha_{1}>0$ is arbitrary and $\beta_{1}, \alpha_{i}, \beta_{i},(i=2, \ldots, k)$ are given by (2.7). Now we observe that $\left(\bar{u}_{1}, \bar{u}_{2}, \ldots, \bar{u}_{k-1}, \bar{u}_{k}\right)$ is a supersolution of (1.1)-(1.3) as long as $\bar{u}_{1}(0, t) \geq 1$. This can be done by choosing $\tau$ large enough. This also allows to assume $\bar{u}_{i}(x, 0) \geq u_{0 i}(x)(i=1,2, \ldots, k)$. Then, by the comparison Lemma 3.1, we obtain that every solution is global.

Now we construct subsolutions with finite time blow-up.

Lemma 4.2. Let $\prod_{l=1}^{k}\left(1+p_{l}\right)<\prod_{l=1}^{k} 2 q_{l}$ (i.e. $\left.\operatorname{det} A<0\right)$, then there exist compactly supported functions $g_{i}(i=1,2, \ldots, k)$, such that

$$
\underline{u}_{i}(x, t)=(T-t)^{\alpha_{i}} g_{i}\left(\xi_{i}\right), \quad \xi_{i}=x(T-t)^{-\beta_{i}}, \quad i=1,2, \ldots, k,
$$

is a subsolution of (1.1)-(1.2).

Proof. To satisfy (3.2) and (3.3), we need that

$$
\begin{gathered}
\left(g_{i}^{p_{i}}\right)^{\prime \prime}\left(\xi_{i}\right) \geq-\alpha_{i} g_{i}\left(\xi_{i}\right)+\beta_{i} \xi_{i} g_{i}^{\prime}\left(\xi_{i}\right), \quad i=1,2, \ldots, k, \\
-\left(g_{i}^{p_{i}}\right)^{\prime}(0) \leq g_{i+1}^{q_{i+1}}(0), \quad i=1,2, \ldots, k, \quad q_{k+1}=q_{1}, \quad g_{k+1}=g_{1} .
\end{gathered}
$$

We choose

$$
g_{i}\left(\xi_{i}\right)=A_{i}\left(a_{i}-\xi_{i}\right)_{+}^{1 /\left(p_{i}-1\right)}, \quad i=1,2, \ldots, k .
$$

Inserting this in the equation, we get

$$
\frac{p_{i}}{\left(p_{i}-1\right)^{2}} A_{i}^{p_{i}-1} \geq-\alpha_{i}\left(a_{i}-\xi_{i}\right)_{+}-\frac{\beta_{i}}{p_{i}-1} \xi_{i} \text { for } 0 \leq \xi_{i} \leq a_{i}, \quad i=1,2, \ldots, k .
$$

Hence, it is enough to impose

$$
\frac{p_{i}}{\left(p_{i}-1\right)^{2}} A_{i}^{p_{i}-1} \geq-\alpha_{i} a_{i}+\frac{\left|\beta_{i}\right|}{p_{i}-1} a_{i}, \quad i=1,2, \ldots, k,
$$

that is

$$
C_{i} A_{i}^{p_{i}-1} \geq a_{i}, \quad i=1,2, \ldots, k .
$$

The boundary conditions impose

$$
\frac{p_{i}}{p_{i}-1} A_{i}^{p_{i}} a_{i}^{1 /\left(p_{i}-1\right)} \geq A_{i+1}^{q_{i+1}} a_{i+1}^{a_{i+1} /\left(p_{i+1}-1\right)}, \quad i=1,2, \ldots, k, A_{k+1}=A_{1}, \quad q_{k+1}=q_{1}, \quad a_{k+1}=a_{1} .
$$

Let

$$
b_{i}=A_{i} a_{i}^{1 /\left(p_{i}-1\right)}, \quad i=1,2, \ldots, k .
$$


Then conditions (4.2) become

$$
\frac{p_{i}}{p_{i}-1} A_{i}^{p_{i}-1} b_{i} \geq b_{i+1}^{q_{i+1}}, \quad i=1,2, \ldots, k, b_{k+1}^{q_{k+1}}=b_{1}^{q_{1}} .
$$

We fix $b_{i}=1(i=1,2, \ldots, k)$ and then $A_{i}$ large enough (and thus $a_{i}$ small) to satisfy (4.1) and (4.3).

Corollary 4.1 Let $\prod_{l=1}^{k}\left(1+p_{l}\right)<\prod_{l=1}^{k} 2 q_{l}$ (i.e. $\left.\operatorname{det} A<0\right)$. Then there exist solutions of (1.1)-(1.3) that blow up in a finite time.

Proof. We only have to apply Lemma 3.1 , to obtain that every solution $\left(u_{1}, \ldots, u_{k}\right)$ that begins above the subsolutions provided by Lemma 4.2 has finite time blow-up.

Lemma 4.3 Let $\prod_{l=1}^{k}\left(1+p_{l}\right)<\prod_{l=1}^{k} 2 q_{l}(i . e . \operatorname{det} A<0)$. If there exists $j(1 \leq j \leq k)$ such that $\alpha_{j}+\beta_{j} \leq 0$, then every nontrivial solution of (1.1)-(1.3) blows up in finite time.

Proof. Without loss of generality, we consider the case $\alpha_{1}+\beta_{1} \leq 0$.

Assume that there exists a global nonnegative nontrivial solution of (1.1)-(1.3), we make the following change of variables

$$
\varphi_{i}\left(\xi_{i}, \tau\right)=(1+t)^{-\alpha_{i}} u_{i}\left(\xi_{i}(1+t)^{\beta_{i}}, t\right), \tau=\log (1+t), \quad i=1,2, \ldots, k .
$$

These functions satisfy

$$
\begin{aligned}
& \varphi_{i \tau}=\left(\varphi_{i}^{p_{i}}\right)_{\xi_{i} \xi_{i}}+\beta_{i} \xi_{i} \varphi_{i \xi_{i}}-\alpha_{i} \varphi_{i}, \quad i=1,2, \ldots, k, \\
& -\left(\varphi_{i}^{p_{i}}\right)_{\xi_{i}}(0, \tau)=\varphi_{i+1}^{q_{i+1}}(0, \tau), \quad i=1,2, \ldots, k, \varphi_{k+1}^{q_{k+1}}=\varphi_{1}^{q_{1}} .
\end{aligned}
$$

As $u_{i}(x, t)(i=1,2, \ldots, k)$ are by hypothesis global, the same is true for $\phi_{i}(i=1,2, \ldots$, $k$,$) . We will construct a solution \left(\widehat{\varphi}_{1}, \ldots, \widehat{\varphi}_{k}\right)$ to system (4.5)-(4.6) increasing with time, with initial data $\left(\widehat{\varphi}_{01}, \ldots, \widehat{\varphi}_{0 k}\right)$ such that $\widehat{\varphi}_{0 i}\left(\xi_{i}\right) \leq u_{i}\left(\xi_{i}, 0\right)(i=1,2, \ldots, k)$. We will prove that $\left(\widehat{\varphi}_{1}, \ldots, \widehat{\varphi}_{k}\right)$ cannot exists globally, thus contradicting the global existence of $\left(u_{1}, \ldots, u_{k}\right)$. In order to achieve our goal, we use an adaptation for systems of the general monotonicity for single quasilinear equation described in [19].

We take initial data $\left(\widehat{\varphi}_{01}, \ldots, \widehat{\varphi}_{0 k}\right)$ satisfying

$$
\left(\widehat{\varphi}_{0 i}^{p_{i}}\right)_{\xi_{i} \xi_{i}}+\beta_{i} \xi_{i}\left(\widehat{\varphi}_{0 i}\right)_{\xi_{i}}-\alpha_{i} \widehat{\varphi}_{0 i} \geq 0, \quad i=1,2, \ldots, k,
$$

and the compatibility conditions

$$
-\left(\widehat{\varphi}_{0 i}^{p_{i}}\right)_{\xi_{i}}(0)=\widehat{\varphi}_{0 i+1}^{q_{i+1}}(0), \quad i=1,2, \ldots, k, \widehat{\varphi}_{0 k+1}^{q_{k+1}}=\widehat{\varphi}_{01}^{q_{1}} .
$$

Hence, arguing as in Lemma 3.2, we have that $\widehat{\varphi}_{i \tau} \geq 0(i=1,2, \ldots, k)$.

Following an idea for scalar equation from [8], we set

$$
\widehat{\varphi}_{01}\left(\xi_{1}\right)=h\left(\xi_{1}+b\right),
$$

where $h$ is the Barenblatt profile

$$
h\left(\xi_{1}\right)=a_{p_{1}}\left(c-\xi_{1}^{2}\right)_{+}^{1 /\left(p_{1}-1\right)} .
$$


Then we have

$$
\begin{aligned}
\left(\widehat{\varphi}_{01}^{p_{1}}\right)_{\xi_{1} \xi_{1}}+\beta_{1} \xi_{1}\left(\widehat{\varphi}_{01}\right)_{\xi_{1}} & -\alpha_{1} \widehat{\varphi}_{01}=-\frac{1}{p_{1}+1} b h_{\xi_{1}}\left(\xi_{1}+b\right) \\
& +\left(\beta_{1}-\frac{1}{p_{1}+1}\right) \xi_{1} h_{\xi_{1}}\left(\xi_{1}+b\right)+\left(-\alpha_{1}-\frac{1}{p_{1}+1}\right) h\left(\xi_{1}+b\right) .
\end{aligned}
$$

The last expression is nonnegative if $\beta_{1}-1 /\left(p_{1}+1\right) \leq 0$ and $-\alpha_{1}-1 /\left(p_{1}+1\right) \geq 0$. But these two conditions are equivalent $\alpha_{1}+\beta_{1} \leq 0$.

Now we take $\widetilde{\alpha}_{i}, \widetilde{\beta}_{i}>0$ such that $\widetilde{\alpha}_{i} \geq \alpha_{i}, \widetilde{\beta}_{i} \geq \beta_{i}(i=1,2, \ldots, k)$. We take as $\widehat{\varphi}_{0 i}$ a solution to

$$
\left(\widehat{\varphi}_{0 i}^{p_{i}}\right)^{\prime \prime}=-\widetilde{\beta}_{i} \xi_{i} \widehat{\varphi}_{0 i}^{\prime}+\widetilde{\alpha}_{i} \widehat{\varphi}_{0 i}, \quad i=1,2, \ldots, k .
$$

There is one-parameter family of solution to this equation (see Theorem 2.4), with $\widehat{\varphi}_{0 i} \geq 0, \widehat{\varphi}^{\prime}{ }_{0 i} \leq 0(i=2, \cdots, k)$. Hence,

$$
\left(\widehat{\varphi}_{0 i}^{p_{i}}\right)^{\prime \prime} \geq-\beta_{i} \xi_{i} \widehat{\varphi}_{0 i}^{\prime}+\alpha_{i} \widehat{\varphi}_{0 i}, \quad i=2, \ldots, k .
$$

Moreover,

$$
\widehat{\varphi}_{0 i}(0)=U_{i,}\left(\widehat{\varphi}_{0 i}^{p_{i}}\right)^{\prime}(0)=U_{i}^{\left(p_{i}+1\right) / 2} V_{* i,} \quad i=2, \ldots, k,
$$

where $V_{*_{i}}<0$ is a constant and $U_{i}$ is the free parameter.

We still have to control the boundary conditions. In order to do this, we choose the constants $c, b$ and $U_{i}(i=2, \ldots, k)$ conveniently. They have to satisfy

$$
\begin{gathered}
\frac{2 p_{1} a_{p_{1}}}{p_{1}-1} b\left(c-b^{2}\right)^{1 /\left(p_{1}-1\right)}=U_{2}^{q_{2}}, b \in\left(0, c^{1 / 2}\right), \\
-V_{* i} U_{i}^{\left(p_{i}+1\right) / 2}=a_{p_{1}}^{q_{i+1}}\left(c-b^{2}\right)^{q_{i+1} /\left(p_{1}-1\right)}, i=2, \ldots, k .
\end{gathered}
$$

Thus, we choose

$$
\begin{aligned}
& U_{2}=c_{2} b^{2 q_{3} /\left(2 q_{2} q_{3}-p_{2}-1\right)}, \quad U_{i}=c_{i} b^{2\left(p_{2}-1\right) q_{i+1}} /\left(\left(p_{i}+1\right)\left(2 q_{2} q_{3}-p_{2}-1\right)\right),\left(i=3, \ldots, k, q_{k+1}=q_{1}\right), \\
& c=b^{2}+\gamma b^{\left(p_{1}-1\right)\left(p_{2}-1\right) /\left(2 q_{2} q_{3}-p_{2}-1\right)},
\end{aligned}
$$

where $c_{i}(i=2, \ldots, k)$ and $\gamma$ are positive constants. Taking $b$ small enough, the initial data $\left(\widehat{\varphi}_{01}, \ldots, \widehat{\varphi}_{0 k}\right)$ is below $\left(u_{1}\left(\xi_{1}, 0\right), \ldots, u_{k}\left(\xi_{k}, 0\right)\right)$. This can be done as $u_{0 i}(i=1,2, \ldots k)$ can be assumed to be positive at the origin.

To conclude the proof, we will show that $\left(\widehat{\varphi}_{1}, \ldots, \widehat{\varphi}_{k}\right)$ converge to a self-similar profile that does not exist in this range of parameters.

Lemma 4.4. There exists $j(1 \leq j \leq k)$ such that

$$
\lim _{\tau \rightarrow \infty} \widehat{\varphi}_{j}\left(\xi_{j}, \tau\right)=\widetilde{\varphi}_{j}\left(\xi_{j}\right)<\infty, \forall \xi_{j}>0 .
$$

Proof. It is clear that $\widehat{\varphi}_{i \xi_{i}} \leq 0(i=1,2, \ldots, k)$. Let us suppose that

$$
\widehat{\varphi}_{i}\left(\xi_{i}, \tau\right) \rightarrow \infty \text { uniformly in }\left(0, \xi_{i 0}\right), \quad i=1,2, \ldots, k .
$$

In the original variables $\left(\widehat{u}_{1}, \ldots, \widehat{u}_{k}\right)$, we have that for any $M>0$ there is a value such that

$$
\left(1+t_{0}\right)^{\alpha_{i}} M \leq \widehat{u}_{i}\left(x, t_{0}\right) \text { for } 0<x\left(1+t_{0}\right)^{-\beta_{i}}<\xi_{i 0}, i=1,2, \cdots, k .
$$


Now we will check that, under these conditions, we can put one of the blowing up subsolutions constructed in Lemma 4.2 below these data. This would lead to a contradiction, as $\left(\widehat{u}_{1}, \ldots, \widehat{u}_{k}\right)$ is global. In order to do this, we need

$$
\begin{aligned}
& \left(1+t_{0}\right)^{a_{1}} M \geq A_{1} a_{1}^{1 /\left(p_{1}-1\right)} T^{\alpha_{1}}, \\
& \xi_{10}\left(1+t_{0}\right)^{\beta_{1}} \geq a_{1} T^{\beta_{1}} .
\end{aligned}
$$

The first equation says that the height at $x=0$ of $\widehat{u}_{1}$ is bigger than that of $\underline{u}_{1}$, and the second says that the support of $\widehat{u}_{1}$ is bigger than the support of $\underline{u}_{1}$. Imposing analogous conditions for $\widehat{u}_{i}$ and $\underline{u}_{i}(i=2, \ldots, k)$ we get

$$
\begin{aligned}
& \left(1+t_{0}\right)^{a_{i}} M \geq A_{i} a_{i}^{1 /\left(p_{i}-1\right)} T^{\alpha_{i}}, \\
& \xi_{i 0}\left(1+t_{0}\right)^{\beta_{i}} \geq a_{i} T^{\beta_{i}} .
\end{aligned}
$$

Taking $T=1+t_{0}$, then $a_{i}$ small enough and $A_{i}$ large enough $(i=1,2, \ldots, k)$, and then $M$ large, then the $2 k$ conditions (4.9)-(4.10) are fulfilled.

Let us remark this parametric evolution comparison method to prove global nonexistence for arbitrary data first introduced in [20], for scalar quasilinear heat equation.

End of the proof of Lemma 4.3. Let us assume that (4.7) holds. Using standard arguments, see [8], we may pass to the limit to obtain that

$$
\left(\widetilde{\varphi}_{1}^{p_{1}}\right)_{\xi_{1} \xi_{1}}+\beta_{1} \xi_{1} \widetilde{\varphi}_{1 \xi_{1}}-\alpha_{1} \widetilde{\varphi}_{1}=0 .
$$

Let $z=\widetilde{\varphi}_{1}^{p_{1}}$, then

$$
z_{\xi_{1} \xi_{1}}+\frac{\beta_{1}}{p_{1}} \xi_{1} z^{\left(1-p_{1}\right) / p_{1}} z_{\xi_{1}} \leq 0
$$

Hence, in $\left(0, \xi_{10}\right), z \geq c>0$,

$$
z_{\xi_{1} \xi_{1}} \leq C z_{\xi_{1}} \text {. }
$$

We conclude that $z$ and therefore $\widetilde{\varphi}_{1}$ cannot be unbounded at $\xi_{1}=0$. In particular, $0<\widetilde{\varphi}_{1}(0) \leq C$. Then, considering the regularity of $\widetilde{\varphi}_{1}$ in the region where $\widetilde{\varphi}_{1}>0$, we can pass to the limit in the boundary condition for $\left(\widehat{\varphi}_{1}^{p_{1}}\right)_{\xi_{1}}$ to obtain that

$$
-\left(\widetilde{\varphi}_{1}^{p_{1}}\right)_{\xi_{1}}(0)=\widetilde{\varphi}_{2}^{q_{2}}(0)
$$

However, as $\alpha_{1}+\beta_{1} \leq 0$, problem (4.11)-(4.12) does not have a nontrivial solution, see Theorem 2.4.

If (4.7) holds for some $j>1$, we can proceed as before to obtain that $\widetilde{\varphi}_{j}(0)<\infty$. Thus, we can pass to the limit in the boundary condition for $\widehat{\varphi}_{j}$, obtaining

$$
-\left(\widetilde{\varphi}_{j}^{p_{j}}\right)_{\xi_{j}}(0)=\widetilde{\varphi}_{j+1}^{q_{j+1}}(0) \text {. }
$$

As $\widetilde{\varphi}_{j+1}(0) \geq \widetilde{\varphi}_{j+1}\left(\xi_{j+1}\right)$, this implies that $\widetilde{\varphi}_{j+1}$ is finite for every $\xi_{j+1} \geq 0$. We get the same contradiction as before. 


\section{Authors' contributions}

The authors declare that the work was realized in collaboration with the same responsibility. All authors read and approved the final manuscript.

\section{Competing interests}

The authors declare that they have no competing interests.

Received: 1 May 2011 Accepted: 19 August 2011 Published: 19 August 2011

\section{References}

1. Pao, CV: Nonlinear Parabolic and Elliptic Equations. Plenum, New York (1992)

2. Fujita, H: On the blowing up of solutions for the Cauchy problem for $u_{t}=\Delta u+u^{1+\alpha}$. J Fac Sci Univ Tokyo Sec IA Math. 16, 105-113 (1996)

3. Levine, HA: The role of critical exponents in blow up theorems. SIAM Rev. 32, 262-288 (1990). doi:10.1137/1032046

4. Andreucci, D, Herrero, MA, Velázquez, JJL: Liouville theorems and blow-up behaviour in a semilinear reaction diffusion systems. Ann Inst H Poincaré Anal Nonlinéaire. 14, 1-53 (1997)

5. Escobedo, M, Levine, HA: Critical blow-up and global existence numbers for a weakly coupled system of reactiondiffusion equations. Arch Rational Mech Anal. 129, 47-100 (1995). doi:10.1007/BF00375126

6. Rossi, JD, Wolanski, N: Blow-up vs. global existence for a semilinear reaction-diffusion system in a bounded domain. Comm Partial Differential Equations. 20, 1991-2004 (1995). doi:10.1080/03605309508821160

7. Wang, $\mathrm{S}, \mathrm{Xie}, \mathrm{CH}$, Wang, MX: Note on critical exponents for a system of equations coupled in the boundary conditions. J Math Anal Appl. 218, 313-324 (1998). doi:10.1006/jmaa.1997.5751

8. Galaktionov, VA, Levine, HA: On critical Fujita exponents for heat equations with nonlinear flux boundary conditions on the boundary. Israel J Math. 94, 1250-146 (1996)

9. Lin, ZG: Blowup behaviors for diffusion system coupled though nonlinear boundary conditions in a half space. Sci China Ser A Math. 47, 72-82 (2004)

10. Pedersen, M, Lin, ZG: Blow-up analysis for a system of heat equations coupled through a nonlinear boundary condition. Appl Math Lett. 14, 171-176 (2001). doi:10.1016/S0893-9659(00)00131-2

11. Wang, MX: The blow-up rates for systems of heat equations with nonlinear boundary conditions. Sci China Ser A Math. 46, 169-175 (2003). doi:10.1360/03ys9018

12. Quirós, F, Rossi, JD: Blow-up sets and Fujita type curves for a degenerate parabolic system with nonlinear boundary conditions. Indiana Univ Math J. 50, 629-654 (2001)

13. Gilding, BH: On a class of similarity solutions of the porous media equation III. J Math Anal Appl. 77, 381-402 (1980). doi:10.1016/0022-247X(80)90234-6

14. Gilding, BH, Peletier, LA: On a class of similarity solutions of the porous media equation. J Math Anal Appl. 55, 351-364 (1976). doi:10.1016/0022-247X(76)90166-9

15. Gilding, BH, Peletier, LA: On a class of similarity solutions of the porous medium equation II. J Math Anal Appl. 57, 522-538 (1977). doi:10.1016/0022-247X(77)90244-X

16. Bénilan, Ph, Cortázar, C, Elgueta, M: Uniqueness and non uniqueness of the solutions of a mixed boundary value problem for the porous medium equation. Rev Un Mat Argentina. 37, 10-15 (1991)

17. Cortázar, C, Elgueta, M, Vázquez, JL: Diffusivity determination in non linear diffusion. Eur J Appl Math. 2, 159-169 (1991). doi:10.1017/S0956792500000450

18. Kalashnikov, AS: Some problems of the qualitative theory of nonlinear degenerate parabolic equations of second order. Uspekhi Mat Nauk 42, 135-176 (1987). (Russian): Russian Math. Surv. 42, 169-222 (1987) (English transl.)

19. Galaktionov, VA: Blow-up for quasilinear heat equations with critical Fujita's exponents. Proc R Soc Edinburg Sect A. $124,517-525$ (1994)

20. Galaktionov, VA, Kurdyumov, SP, mikhailov, AP, Samarskii, AA: Unbounded solutions of the cauchy problem for the parabolic equation $u_{t}=\mathbb{\nabla}\left(u^{\circ} \otimes u\right)+u^{\beta}$. Dokl Akad Nauk SSSR Ser Math Phys 252, 1362-1364 (1980). (Russian); Soviet Phys. Dokl. 25, 458-459 (1980) (English transl.)

doi:10.1186/1687-2770-2011-15

Cite this article as: $\mathrm{Xu}$ and Song: Critical parameter equations for degenerate parabolic equations coupled via nonlinear boundary flux. Boundary Value Problems 2011 2011:15.

\section{Submit your manuscript to a SpringerOpen ${ }^{\circ}$ journal and benefit from:}

- Convenient online submission

- Rigorous peer review

- Immediate publication on acceptance

- Open access: articles freely available online

- High visibility within the field

- Retaining the copyright to your article

Submit your next manuscript at $\boldsymbol{s p r i n g e r o p e n . c o m ~}$ 